\title{
Control automático del material crudo en una fábrica de cemento con analizador de rayos $X$ y ordenador de proceso $(*)$
}

\author{
JUAN PUIG MONTRAVeta, Licenciado en Ciencias Quimicas \\ Jefe de Laboratorio de CEMENTOS MOLINS, S. A. \\ Barcelona (ESPAÑA)
}

\begin{abstract}
En el presente trabajo se describen los equipos utilizados en CEMENTOS MOLINS, S. A., de Barcelona, España, en el control automático del crudo de cemento, su puesta en marcha, los problemas que han surgido durante los dos primeros años de funcionamiento, y cuáles han sido los resultados conseguidos.
\end{abstract}

En un futuro inmediato se realizará un detenido estudio acerca de la exactitud de resultados y el tiempo necesario para la preparación de muestras para el análisis por fluorescencia de rayos $X$ de crudo de cemento preparadas con la técnica de la perla de bórax o bien con la técnica del empastillado. $Y$, a continuación, se extenderá la técnica de análisis por rayos $X$, con la preparación de muestras más adecuada para todos los materiales comúnmente analizados en una fábrica de cemento: calizas, arcillas, areniscas, piritas, bauxitas, cementos, yesos, etc.

\section{GENERALIDADES}

\section{Introducción}

Las cada vez mayores capacidades de producción de las modernas fábricas de cemento, así como la necesidad de una más alta y uniforme calidad del producto acabado, exigen una automatización del proceso y un control de calidad exacto y rápido, tanto en la obtención de los resultados de análisis como en la realización de los cambios oportunos. El logro más completo de dicho grado de control de calidad se puede conseguir hoy día con ayuda de un analizador de fluorescencia de rayos $\mathrm{X}$ y un ordenador de proceso.

(*) Trabajo presentado en el "IX Colloque International sur l'analyse de la matière", celebrado en Toulouse, octubre 1972 . 
El cemento portland es un material obtenido por molienda conjunta, a un grado de finura adecuado, de clínker portland con una pequeña proporción de piedra de yeso. El clínker portland, que es una roca o piedra artificial constituida mineralógicamente por silicatos y aluminatos cálcicos, como principales constituyentes, se obtiene por calcinación del material crudo, realizada, en nuestro caso, en hornos rotatorios con recuperadores de calor. El material crudo se obtiene por molienda y homogeneización de materias primas ricas en cal, sílice, alúmina y óxido de hierro, debiendo alcanzar dicho crudo una granulometría y composición química prefijadas y constantes. Como materias primas utilizamos en nuestra fábrica las siguientes: caliza, arcilla, arenisca y cenizas de pirita tostada.

El control del crudo es el primer y más decisivo paso, a la vez que el más complejo, para garantizar la calidad y regularidad del cemento a obtener.

Hasta 1970 no disponíamos de más medio de control que la titulación en carbonato del material crudo, con corrección manual y subjetiva de las proporciones de materias primas. El análisis químico completo, por vía tradicional, exigía unas 8 horas, y por ello no podía utilizarse para un control continuo de la molienda de crudo.

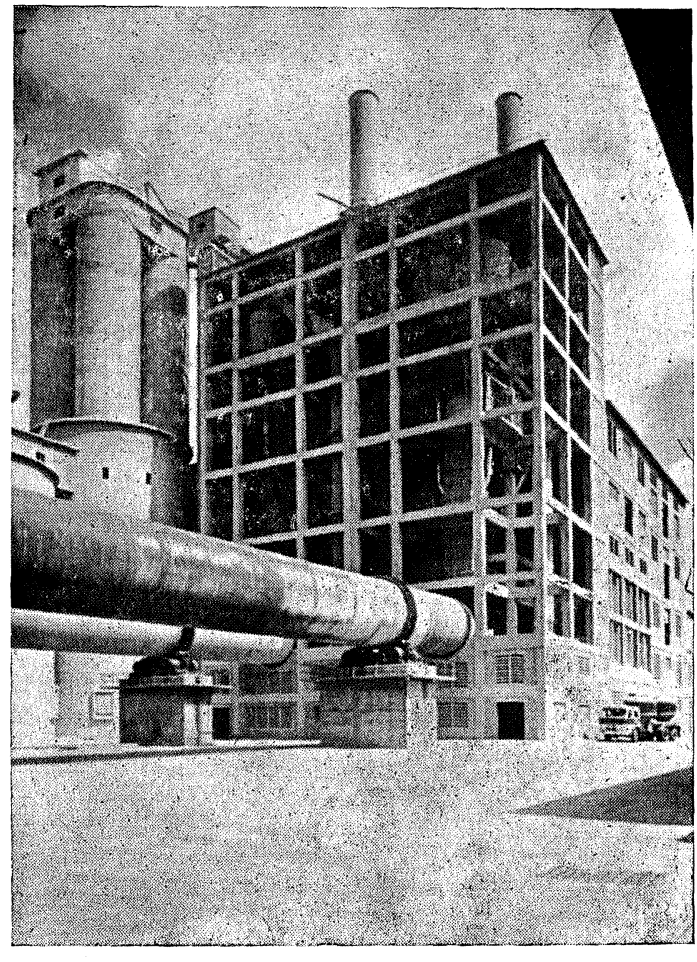

Vista general de la fábrica.
A partir de 1970, el control del crudo en Cementos Molíns, S. A., se realiza mediante análisis por fluorescencia de rayos $\mathrm{X}$ de muestras preparadas en forma de perlas, con interpretación de resultados y corrección automática de las básculas dosificadoras de materias primas llevadas a cabo por un ordenador de proceso.

En la química del cemento portland, los análisis de crudo, clínker y cemento, se limitan a darnos los contenidos de los óxidos fundamentales $\left(\mathrm{CaO}, \mathrm{SiO}_{2}, \mathrm{Al}_{2} \mathrm{O}_{3}, \mathrm{Fe}_{2} \mathrm{O}_{3}\right.$ y $\left.\mathrm{MgO}\right)$, si bien para definirlos mejor se calculan sus módulos o su composición mineralógica potencial. En nuestra fábrica se han fijado unos valores ideales de los módulos de saturación en cal, de sílice y de alúmina, y a los cuales tienden crudo, clínker y cemento.

Módulo o grado de saturación en cal:

$$
\text { Sat. } \mathrm{Cal}=\frac{\mathrm{C}}{2,80 \cdot \mathrm{S}+1,18 \cdot \mathrm{A}+0,65 \cdot \mathrm{F}}
$$

Módulo de sílice o silícico:

$$
\text { M. Silíc. }=\frac{S}{A+F}
$$


Módulo de alúmina o alumínico:

$$
\text { M. Alúm. }=\frac{\mathrm{A}}{\mathrm{F}}
$$

Siendo:

$$
\mathrm{C}=\% \mathrm{CaO} ; \quad \mathrm{S}=\% \mathrm{SiO}_{2} ; \quad \mathrm{A}=\% \mathrm{Al}_{2} \mathrm{O}_{3} \text { y } \mathrm{F}=\% \mathrm{Fe}_{2} \mathrm{O}_{3} .
$$

\section{Descripción del equipo de control}

Máquina semiautomática de preparación de perlas

Dicha máquina, marca Philips, modelo PW 1234, es un dispositivo que prepara semiautomáticamente perlas para ser empleadas en el análisis por fluorescencia de rayos $\mathrm{X}$ de productos pulverulentos como crudos, cementos, escorias, bauxitas, etc. Permite su manipulación por personal no especializado y es adecuada para llevar el control continuo de un proceso de fabricación.

La hemos simplificado en parte, al suprimirle los dispositivos de pesada automática de muestra y de bórax, por considerar que, además de poseer escasa exactitud, el tiempo que requiere la limpieza del embudo dosificador de material a analizar, entre muestra y muestra, es prácticamente igual o mayor que el necesario para pesar la muestra y el bórax en una moderna balanza analítica monoplato.

El crudo, previamente pesado, se vierte sobre un crisol de aleación de platino y se calienta por inducción de alta frecuencia, calcinándose la muestra y perdiendo su anhídrido carbónico y su agua de cristalización, a la vez que se oxidan los elementos metálicos que podrían pasar al crisol durante la etapa de fusión, acortando su vida.

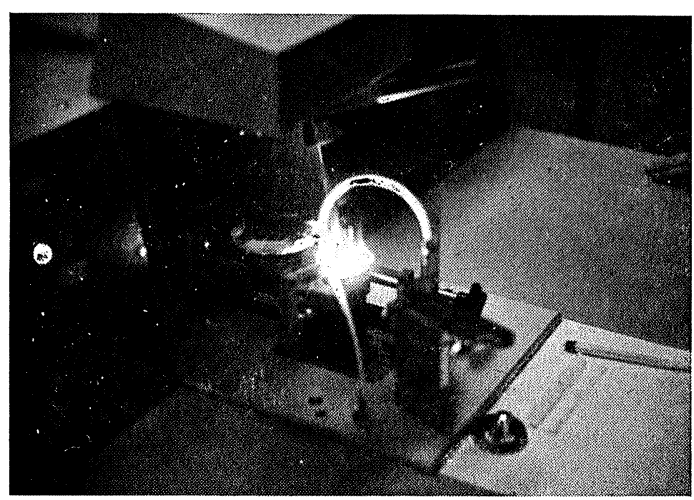

Máquina de perlas fundiendo.

A continuación se añade el bórax, ya pesado, y se inicia la fusión, calentando por el mismo sistema hasta conseguir un vidrio homogéneo que al enfriarse se convierte en una perla de bórax, apta para ser analizada. Para lograr que la perla se despegue del crisol, y así, entera, se pueda analizar, durante su enfriamiento se somete a un chisporroteo por alta tensión.

Mediante un sistema de vacío se retira la perla, aún caliente, del crisol y se coloca en el portamuestras para ser analizada.

Equipo multicanal de fluorescencia de rayos $X$

Dicho equipo, marca Philips, modelo PW 1270, es capaz de analizar simultáneamente hasta 14 elementos, siendo su funcionamiento muy simple. En la actualidad analizamos 5 elementos: $\mathrm{Ca}, \mathrm{Fe}, \mathrm{Al}, \mathrm{Si}$ y $\mathrm{Mg}$, y esperamos en un futuro inmediato analizar además $\mathrm{S}$ y K. 
La potencia del tubo emisor de rayos $\mathrm{X}$ es de $2,7 \mathrm{~kW}$, si bien trabajamos a $50 \mathrm{kV}$ y 50 $\mathrm{mA}$, es decir con un consumo de $2,5 \mathrm{~kW}$.

El espectrómetro está preparado para poder trabajar al aire, en atmósfera de helio y en vacío. Utilizamos la última técnica, con un vacío de 0,05 a $0,1 \mathrm{~mm}$ de $\mathrm{Hg}$ o torr.

Permite varias formas de análisis: métodos absoluto, monitor, relativo y relativo teniendo en cuenta el ruido de fondo. Usamos el relativo, con respecto a una muestrapatrón de vidrio. Con dicho método, la muestra-patrón está bajo rayos $\mathrm{X}$ al introducir la muestra a analizar, y al pulsar el botón de análisis (Start) se inicia inmediatamente el análisis de la muestra-patrón de vidrio hasta alcanzar las cuentas prefijadas para cada uno de los canales o elementos a analizar, a la vez que coloca bajo vacío a la muestra objeto de análisis. Contabilizadas todas las cuentas prefijadas, gira automáticamente el carrusel con los portamuestras, colocando la muestra a analizar bajo rayos $\mathrm{X}$ y procediendo a determinar las cuentas de cada elemento a analizar o canal durante el mismo tiempo que invirtió para totalizar las cuentas prefijadas en la patrón de vidrio. En estas condiciones, no aparecen variaciones en las curvas de calibración motivadas por envejecimiento del tubo de rayos $\mathrm{X}$ con el tiempo. Utilizando dicho método relativo de análisis, la duración total del mismo es de unos 3 a 4 minutos.

$\mathrm{El}$ equipo de rayos $\mathrm{X}$ suele poseer un pequeño procesador de datos, capaz de transformar las cuentas o impulsos en concentraciones, con posibilidad de analizar hasta tres tipos distintos de materiales. Si el analizador está conectado a un gran ordenador de proceso, como en nuestro caso, se suele prescindir del procesador de datos y el ordenador recibe directamente las cuentas de cualquier tipo de material, cuya calibración se haya realizado previamente, mediante la adopción de un adecuado código de identificación de muestra. A tal fin, se dispone de cinco dígitos que permiten definir la muestra analizada, y que se seleccionan a voluntad exteriormente. Hemos dedicado los dos primeros dígitos del número de muestra como código del material, pudiéndose analizar hasta 99 materiales distintos o análogos y con distintos tratamientos posteriores por el ordenador de proceso.

El equipo analizador dispone de un sistema automático de corrección del tiempo muerto, un regulador de la presión del gas en los detectores proporcionales de flujo y una unidad de estabilización de temperatura en la cámara de cristales.

La salida de los resultados de análisis se realiza mediante una máquina de escribir IBM, si bien se dispone, además, de una conexión o "interface" que permite al ordenador recibir directamente los datos o cuentas del analizador.

\section{Ordenador de proceso}

Para llevar el control de calidad del material crudo, así como para automatizar el proceso y realizar además tareas administrativas, está instalado junto al equipo analizador un ordenador de proceso, marca IBM, modelo 1800, que posee las siguientes partes esenciales:

- Una unidad central, con memoria de $16 \mathrm{~K}$, ampliable.

- Una unidad de almacenamiento de discos, con tres cabezas lectoras-grabadoras, de las cuales dos son para ser utilizadas en el control del proceso y una para trabajos administrativos. La capacidad de almacenamiento de cada disco es de $500 \mathrm{~K}$.

- Una máquina de escribir para entradas y salidas de datos del proceso, y dos impresoras para salidas de resultados. 
- Una impresora rápida para la realización de trabajos administrativos.

- Una perforadora, una lectora perforadora, y una verificadora de fichas.

- Dispositivos de recepción de hasta 200 señales analógicas, y para entrada-salida de unas 100 señales digitales. Los correspondientes convertidores analógicos digitales. Ambos dispositivos son ampliables, para permitir alcanzar una mayor o total automatización del control del proceso.

- Consola con teclado funcional, teclado numérico, registrador múltiple de curvas y display-digital (tipo Nixe). Permitirá, al operario de la sala de control centralizado, conocer el valor de las variables conectadas al ordenador, modificar parámetros de los sistemas de control y poner o quitar diferentes bucles de fases del proceso bajo el control del ordenador.

El sistema operativo del ordenador es el TSX, Time Sharing Executive Systeme, o Sistema Ejecutivo de Trabajo Compartido, lo que permite simultanear trabajos de proceso y administrativos, con preferencia de los primeros.

El lenguaje habitual utilizado es el FORTRAN, siendo posible utilizar asimismo el ASSEMBLER.

\section{PUESTA EN MARCHA DEL SISTEMA DE CONTROL}

\section{Puesta en marcha y calibración del equipo de análisis}

Se seleccionan inicialmente 13 muestras de crudo, con las que se cubría ampliamente toda la posible variación de dicho material. Dichas muestras, previamente homogeneizadas, se remitieron a 4 distintos laboratorios para que procedieran a su análisis. Dos de

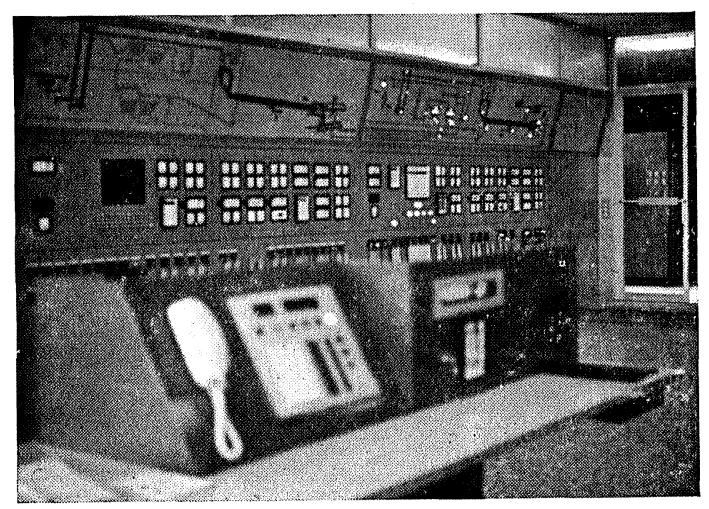

Consola de la sala de control centralizado. dichos laboratorios eran oficiales, otro era de una empresa danesa suministradora de maquinaria, y el cuarto era el de Cementos Molíns, S. A. Pensábamos considerar la media de los resultados analíticos de estos laboratorios como el análisis químico real de dichas muestras. Pero los resultados presentaban una dispersión tan elevada, que era imposible considerar la media de ellos. Se tomó como análisis químico representativo de las muestras de calibración, la media de 4 análisis realizados en nuestro propio laboratorio por analistas diferentes; y ello por dos motivos: primero, por ser los que presentaban menor dispersión, y segundo, por considerar que en caso de obtener, en cualquier ocasión, resultados anómalos con el equipo analizador de rayos $\mathrm{X}$, siempre dichos resultados se contrastan frente a análisis químicos tradicionales, y que cualquier laboratorio extraño a la fábrica, oficial o no, requiere a veces meses para realizar los análisis que se le solicitan, mientras que en el propio laboratorio de fábrica ello requiere, a lo sumo, unos pocos días. 
Si bien todas las muestras analizadas de la molienda de crudo estaban dentro del campo cubierto por las muestras de calibración, se consideró oportuno ampliar el número de ellas, eligiéndolas de entre aquellas que estaban más cercanas a los valores de consigna. Por ello, en la actualidad, disponemos de unas 20 muestras para la calibración de crudo.

Una vez ya dibujadas las curvas que relacionaban las intensidades de rayos $\mathrm{X}$, como impulsos o cuentas, con las concentraciones, se determinó la ecuación de la curva a la que más se aproximan los datos que la definen mediante un sistema de mínimos cuadrados.

La ecuación más completa que relaciona concentraciones con intensidades es la siguiente:

$$
\left.\mathrm{C}_{\mathrm{i}}^{\mathrm{x}}=\left[\frac{\frac{\mathrm{I}_{\mathrm{i}}^{\mathrm{x}}}{\mathrm{I}_{\mathrm{i}}^{\mathrm{s}}} \cdot \frac{\mathrm{T}_{\mathrm{i}}^{\mathrm{s}}-\mathrm{I}_{1}^{\mathrm{s}} \cdot \mathrm{t}}{\mathrm{T}_{\mathrm{i}}^{\mathrm{x}} \cdot \mathrm{I}_{\mathrm{i}}^{\mathrm{x}} \cdot \mathrm{t}}-\mathrm{K}_{\mathrm{i}}^{\mathrm{x}}}{(1)}\right] \mathrm{K}_{\mathrm{i}}^{\mathrm{x}}\right] \cdot \mathrm{C}_{\mathrm{i}}^{\mathrm{s}} \cdot\left[1+\mathrm{K}_{\mathrm{ij}}\left(\mathrm{C}_{\mathrm{j}}{ }^{\mathrm{s}}-\mathrm{C}_{\mathrm{j}}{ }^{\mathrm{x}}\right)\right]
$$

en donde:

(1) concentración en la muestra analizada.

(2) relación de intensidades, entre la muestra analizada y la patrón.

(3) corrección por tiempo muerto.

(4) corrección por ruido de fondo o bakground.

(5) concentración del elemento analizado en la muestra-patrón.

(6) corrección por efectos matrices.

En el equipo PW 1270 ya existe una corrección automática del tiempo muerto, por lo cual la corrección (3) no es necesaria. Al trabajar con perlas, la corrección (6) de efectos matrices también es innecesaria. Además, es desconocido el análisis de la muestrapatrón de vidrio $\left(\mathrm{C}_{\mathrm{i}}^{\mathrm{s}}\right)$.

Esta ecuación no es válida para ser aplicada en nuestro método de análisis, el relativo, por desconocer cuál es el análisis exacto de la muestra patrón de vidrio, y desconocer pues los valores de las $\mathrm{C}_{\mathrm{i}}$ s. Por ello debimos recurrir a la calibración frente a una serie de muestras de análisis perfectamente conocido.

Con dichas simplificaciones, la fórmula que relaciona intensidades con concentraciones puede expresarse así:

$$
C_{i}=I_{i}\left(m_{i}+\sum_{j=1}^{j=n} m_{i j} \cdot I_{j}\right)+\left(b_{i}+\sum_{j=1}^{j=n} b_{i j} \cdot I_{j}\right) ;
$$

en la cual:

$\mathrm{C}_{\mathrm{i}}=$ concentración del elemento i.

$\mathrm{I}_{\mathrm{i}} \quad=$ intensidad del elemento $\mathrm{i}$.

$I_{j}=$ intensidad del elemento $j$.

$\mathrm{m}_{\mathrm{i}}=$ pendiente principal de concentración.

$b_{i}=$ concentración del fondo, o background, del elemento i.

$b_{i j}=$ factor de influencia de la intensidad $I_{j}$ en el cambio de valor de la ordenada en el origen $b_{i}$. 
Esta fórmula [II] puede simplificarse en tres particulares casos:

a) $\mathrm{Si}$ debe tenerse en cuenta una corrección por un efecto interelemental:

$$
C_{i}=I_{i}\left(m_{i}+m_{i j} \cdot I_{j}\right)+b_{i} .
$$

b) Para realizar una calibración en forma de curva:

$$
\mathrm{C}_{\mathrm{i}}=\mathrm{I}_{\mathrm{i}}\left(\mathrm{m}_{\mathrm{i}}+\mathrm{m}_{\mathrm{ij}} \cdot \mathrm{I}_{\mathrm{i}}\right)+\mathrm{b}_{\mathrm{i}} .
$$

c) Siendo una recta la curva de calibración:

$$
\mathrm{C}_{\mathrm{i}}=\mathrm{m}_{\mathrm{i}} \cdot \mathrm{I}_{\mathrm{i}}+\mathrm{b}_{\mathrm{i}} .
$$

La que hemos utilizado es [V] en expresión relativa, con cociente respecto a concentración e intensidades del elemento mayoritario, que, en el caso de análisis de crudos, clínkeres y cementos, es la cal. Las ecuaciones de las rectas de calibrado han sido pues del tipo:

$$
\frac{\mathrm{C}_{\mathrm{i}}}{\mathrm{C}_{\mathrm{Ca} 0}}=\mathrm{A} \cdot \frac{\mathrm{I}_{\mathrm{i}}}{\mathrm{I}_{\mathrm{Ca} 0}}+\mathrm{B} .
$$

Utilizando este método, relativo con respecto al elemento mayoritario, se consiguen las siguientes ventajas analíticas:

1. ${ }^{a} \quad$ Evita errores de dilución como:

a) Inexactitud en la pesada de muestra o bórax.

b) Variaciones en la temperatura de fusión, siempre que se sobrepase un valor mínimo de temperatura.

c) Variaciones en la duración del tiempo de fusión.

d) Posibles pegaduras de trozos de perla en el crisol.

e) Posibles salpicaduras de material fundido, por calentamiento demasiado rápido.

f) El tener que refundir la perla, por haberse roto.

2. ${ }^{a} \quad$ Minimiza los errores que se producirían, por no estar perfectamente plana la superficie de la perla.

Cuando se expongan los resultados de reproducibilidad se podrán observar los límites máximos de variaciones, que son automáticamente corregidos al trabajar con este método relativo.

El ajuste de los resultados a esta recta de calibración lo realiza el ordenador por un sistema de mínimos cuadrados, aplicando un programa adecuado. En cada calibración, basta perforar en unas fichas a las intensidades obtenidas con las muestras de calibración y sus análisis químicos, y pasar el programa correspondiente. Se obtiene como resultado la pendiente, la ordenada en el origen y la desviación estándar de las muestras de calibración con respecto a esta recta de calibrado. La forma con que aparecen estos datos por la impresora del ordenador es, por ejemplo, la siguiente:

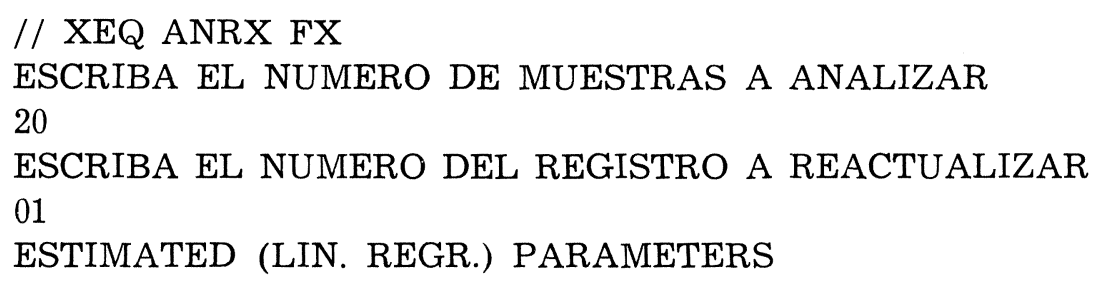




\begin{tabular}{llrrr}
\hline 1 & $(\mathrm{Ca})$ & $0.00000 \mathrm{E} 00$ & $0.00000 \mathrm{E} 00$ & $0.00000 \mathrm{E} 00$ \\
2 & $(\mathrm{Si})$ & $0.71618 \mathrm{E} 01$ & $-0.32299 \mathrm{E}-01$ & $0.33710 \mathrm{E}-02$ \\
3 & $(\mathrm{Al})$ & $0.13191 \mathrm{E} 01$ & $-0.19920 \mathrm{E}-01$ & $0.91946 \mathrm{E}-03$ \\
4 & $(\mathrm{Fe})$ & $0.17080 \mathrm{E} 00$ & $-0.40833 \mathrm{E}-03$ & $0.19782 \mathrm{E}-03$ \\
5 & $(\mathrm{Mg})$ & $-0.57476 \mathrm{E}-03$ & $0.17990 \mathrm{E}-01$ & $0.28911 \mathrm{E}-05$
\end{tabular}

\section{Interpretación de resultados de análisis por el ordenador}

El análisis de las muestras de crudo, por el espectrómetro multicanal de fluorescencia de rayos $X$, se realiza utilizando el método relativo frente a una muestra-patrón de vidrio, en la cual debe contabilizar un prefijado número de impulsos o cuentas, distinto para cada elemento; y tras memorizar el tiempo que ha necesitado en cada canal, lo convierte en tiempo de análisis de la muestra desconocida. En las condiciones actuales de trabajo, los elementos y cuentas prefijadas son:

\begin{tabular}{cccc} 
Canal & Elemento & Cuentas prefijadas & Tiempo de análisis \\
\hline 1 & $\mathrm{Ca}$ & $4 \times 10^{6}$ & $90 \mathrm{~s}$ \\
2 & $\mathrm{Fe}$ & $10^{6}$ & $85 \mathrm{~s}$ \\
3 & $\mathrm{Al}$ & $10^{5}$ & $35 \mathrm{~s}$ \\
4 & $\mathrm{Si}$ & $10^{6}$ & $60 \mathrm{~s}$ \\
5 & $\mathrm{Mg}$ & - & $100 \mathrm{~s}$ \\
& & & (fixed time)
\end{tabular}

Por todo ello, es fácil deducir que el tiempo de análisis supera ligeramente los 3 minutos.

Los resultados de análisis, como cuentas totalizadas, se imprimían en la máquina de escribir IBM del propio espectrómetro. Y, en esta primera fase, se transcribían a un papel para introducirlas manualmente al ordenador, que, poseyendo ya las curvas de calibrado oportunas, transformaba dichas cuentas en concentraciones y módulos.

Si se trataba de llevar el control de la molienda de crudo, se daban manualmente al ordenador las cuentas del analizador y, además, los datos de la muestra que se analizó: hora de toma de muestra, molino de que se retiró y valores de los contadores totalizadores de las básculas que alimentan las materias primas. Mediante una serie de programas adecuados, el ordenador emitía un parte en el que informaba del análisis de la muestra, de cuál era el análisis medio del material en los silos, de los análisis estimativos de las materias primas utilizadas, y ordenaba finalmente cuáles debían ser las proporciones de materias primas a dosificar en la alimentación al molino durante el próximo período de muestra.

\section{Lectura directa por el ordenador de los resultados de análisis}

Un paso más dentro de la automatización del control de crudo fue la lectura directa por el ordenador de los resultados del analizador. Para ello, se creó una conexión, o "interface", entre analizador y ordenador, con el fin de adecuar las tensiones de salida de datos del analizador a las de entradas de datos del ordenador. 
Para tener la seguridad de que las cuentas transmitidas por el analizador son correc a $^{-}$ mente recibidas e interpretadas por el ordenador, las cuentas resultado del análisis son escritas, simultánea y consecutivamente, por las máquinas de escribir del analizador y del ordenador.

Las cuentas se envían al ordenador secuencialmente: primero la codificación del número de muestra, y después las cuentas de los canales $1,2,3,4$ y 5 . Si la transmisión fuera excesivamente rápida, o por cualquier motivo el ordenador no recibiera secuencialmente alguna cuenta o cifra de los resultados transmitidos, interpreta de inmediato que ha habido algún error en la transmisión de datos y ordena al operario que repita el análisis, dándole el siguiente parte de alarma:

\section{"ERROR EN LA TRANSMISION DE DATOS ANALIZADOR-ORDENADOR. VUELVAN A EFECTUAR ANALISIS"}

Este dispositivo que posibilita la lectura directa por el ordenador de los datos de análisis fue conectado y puesto en funcionamiento al cabo de unos 2 meses de la puesta en marcha del equipo analizador, y ha funcionado bien hasta el presente.

\section{Lectura directa, por el ordenador, de los datos del proceso}

Todos los contadores totalizadores de las básculas dosificadoras de materias primas que alimentan a los molinos de crudo, así como los potenciómetros que permiten la modificación de dichas básculas, se hallan emplazados en los cuadros de control de dichos molinos, que están situados en la sala de control centralizado del proceso, sita junto a las salas del espectrómetro y del ordenador.

El paso siguiente en la automatización del control del crudo consistió en la conexión al ordenador de las señales analógicas de dichos contadores, a través de un convertidor analógico-digital. El ordenador toma lecturas instantáneas cada 15 segundos y las promedia e integra cada 2 minutos, pudiendo conocer en cualquier momento la producción del molino, así como las proporciones de las materias primas utilizadas.

Cuando el operario cerraba un contacto, en el momento de la toma de muestra, se informaba el ordenador de la hora y molino del cual se retiraba la muestra, y éste procedía a totalizar la producción y calcular los porcentajes de materias primas consumidos.

En esta etapa intermedia el ordenador leía ya directamente las cuentas o resultados del analizador y los contadores de las básculas dosificadoras, informando, finalmente, de cuáles eran las proporciones oportunas de las materias primas a utilizar en el próximo período de molienda.

\section{Cierre del bucle de control}

Para cerrar el bucle sólo faltaba que el ordenador leyera y modificara los potenciómetros reguladores de las básculas dosificadoras de materias primas. Ello se consiguió al conectar los potenciómetros al ordenador, al cabo de unos 6 meses de la puesta en marcha de la instalación de control. 
Ya desde entonces, una vez el ordenador ha hecho todos los cálculos oportunos, automáticamente manda una señal que conecta durante un cierto período de tiempo los motores reguladores de las básculas dosificadoras de las materias primas, a fin de conseguir que los porcentajes de las mismas sean los que calculara.

Como labor complementaria, el ordenador realiza además un trabajo de supervisión, al comprobar, cada 2 minutos, que las proporciones de las materias primas que se han consumido en dicho intervalo de tiempo son precisamente las que él calculó con anterioridad. En caso contrario, vuelve a modificar las posiciones de los potenciómetros hacia los valores de consigna.

\section{PROBlemas QUE ha PLANTEAdo LA INTROdUCCION DE DICHO SISTEMa DE CONTROL}

\section{Máquina de perlas. Durabilidad de los crisoles}

Su puesta en marcha no presentó dificultades. La durabilidad de los crisoles ha sido la primera preocupación seria que tuvimos. Con el equipo recibimos dos crisoles, y se nos informó que podíamos solicitar más o repararlos a través de una firma extranjera. Cada crisol permitía obtener de 150 a 200 perlas como máximo.

La primera solución, parcial, la conseguimos al hallar una empresa nacional, con talleres en Madrid y Barcelona, dispuesta a fabricarnos crisoles nuevos y repararnos los averiados, según planos y aleación originales. Al tratarse de una aleación especial (85\% $\mathrm{Pt}, 10 \% \mathrm{Rh}$ y $5 \% \mathrm{Au}$ ), que ellos normalmente no preparaban, los plazos de entrega eran muy largos; y si bien inicialmente nos prepararon unos 10-12 crisoles, dicho número se debió duplicar muy pronto. Los plazos de reposición de crisoles usados era de 1 mes como mínimo.

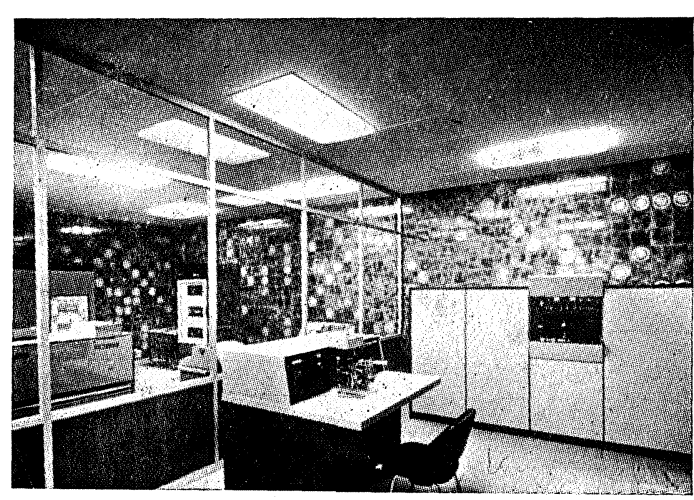

Analizador y ordenador.

La duración de los crisoles era limitada:' 100 a 150 perlas, en el mejor de los casos. La aparición de grietas era la causa más frecuente de averías que obligaba a mandarlos reconstruir, pues por ellas perdía material e impedía obtener perlas enteras. Diariamente se efectúan de 75 a 100 análisis, y por ello un crisol debía mandarse reconstruir después de, como máximo 2 días de uso, si bien cada 40 perlas, o menos, debíamos mandarlo a nuestro laboratorio para limpiarlo y aplanar su fondo. La escasez de crisoles nos hizo en ocasiones reducir la frecuencia de análisis, y en casos extremos estuvimos a punto de tener que abandonar el nuevo método de control para seguir con el tradicional de carbonatos. Ello nos llevó a formalizar un contrato que garantizase un suministro mínimo de 15 crisoles por mes.

Intentamos mejorar la situación buscando otras aleaciones que fueran más durables. Con una de $90 \% \mathrm{Pt}$ y $10 \% \mathrm{Rh}$, construimos unos crisoles que, si bien poseían gran dureza, no permitían utilizarlos, ya que el material fundido tenía en ellos un inadecuado ángulo de mojado, y tendía a salirse. Realizamos ensayos con otros tipos de aleaciones, pero sin éxito. 
Finalmente, con una aleación de $95 \% \mathrm{Pt}$ y $5 \% \mathrm{Au}$, el material fundido se comporta bien y no aparecen grietas. Dicha aleación es muy maleable y los crisoles se deforman con suma facilidad, no sólo en el fondo sino tambien en las alas, con lo cual el contacto con las patas de sujección es deficiente. Para darles forma, y aprovechando su maleabilidad, nos construimos una pequeña prensa manual que nos permite reconstruir por completo a los crisoles deformados. Ahora la durabilidad de los crisoles es, al menos, cinco veces la inicial, permitiendo cada crisol obtener, normalmente, de 800 a 1.000 perlas antes de mandarlo fundir y reconstruir.

El material que se coloca debajo de los crisoles, y encima de la espiral de inducción, es de naturaleza cuarzosa. Puede ser un tejido de lana de cuarzo o bien unos discos del mismo material. El único que realmente funciona es el primero, y con él se trabaja mientras dura la marcha continuada de un mismo crisol, y que suele ser de un máximo de hasta 50 a 70 perlas antes de ser enviado al laboratorio para ser limpiado y reformado; es decir, debe cambiarse cada vez que se utiliza un nuevo crisol. En cambio, los discos de cuarzo, que hemos utilizado en algunas ocasiones, no aguantan más de una o dos perlas consecutivas, pues se rompen con suma facilidad.

El bórax que hemos utilizado ha sido el Merck, ref. 6304, y no ha suscitado ningún problema.

Finalmente, un motivo de distorsión es la variación acusada de la tensión suministrada al equipo. Dicha variación suele ser frecuente en fábricas de cemento, pues al conectar o desconectar grandes molinos, que suelen llegar a consumir varios miles de $\mathrm{kWh}$, la tensión fluctúa a veces en valores que llegan a alcanzar un 7-10\%. Estas variaciones modifican la temperatura de fusión, y ello puede llegar a impedir la consecución de perlas enteras, o a darlas mal fundidas y heterogéneas, obteniendo unos resultados analíticos falsos.

\section{Problemas que ha causado el equipo analizador de rayos $\mathbf{X}$}

Las partes mecánicas de carrusel y vacío han motivado el paro del equipo en varias ocasiones. En cuanto al carrusel, caerse y perderse una muestra ha sido lo más frecuente, si bien también a veces ha quedado atrancado en su movimiento de giro o de presentación de muestra. En el sistema de vacío, aparte de roturas de la ventana de los detectores (recordemos que se usan ventanas de 1 y 6 micras de espesor), ha sido el mal funcionamiento mecánico de las válvulas de vacío el que nos ha ocasionado más transtornos, obligando a varios paros y en una ocasión nos causó la rotura e inutilización de la muestra-patrón de vidrio. En ocasiones, el pulsar inadecuadamente las teclas del panel principal ha producido pérdidas momentáneas de vacío, o atrancos en el carrusel. La disminución de reproducibilidad del equipo, se ha podido atribuir, en un par de ocasiones, a no funcionar el spinner adecuadamente.

Durante largo tiempo obtuvimos resultados esporádicamente anómalos en los canales 3 y 5 , de $\mathrm{Al}$ y $\mathrm{Mg}$. Observamos, como norma, que dos resultados consecutivos que presentaban poca dispersión eran ambos correctos; pero si presentaban mucha dispersión, o ambos eran anómalos o lo era uno de ellos. Mientras no detectamos la avería, decidimos analizar todas las muestras por duplicado.

Para ello programamos al ordenador con el fin de que recibiera todos los análisis por duplicado, promediara los dos resultados, y comparara si cada análisis individual poseía un error menor que el máximo tolerado para cada uno de los elementos analizados. Si 
tal diferencia o error en dos análisis consecutivos era menor que el tolerado, daba como análisis correcto la media de ambos análisis. En caso contrario, emitía un parte que obligaba a repetir el análisis, y que decía:

\section{"LAS CONCENTRACIONES DIFIEREN DEL LIMITE ESTABLECIDO. VUELVA A REPETIR EL ANALISIS"}

Los límites máximos que se establecieron fueron los siguientes:

\begin{tabular}{cccc} 
Elemento & $\begin{array}{c}\text { Error relativo } \\
\text { máximo tolerado }\end{array}$ & $\begin{array}{c}\text { Análisis } \\
\text { medio }\end{array}$ & $\begin{array}{c}\text { Error absoluto } \\
\text { máximo tolerado }\end{array}$ \\
\hline $\mathrm{Ca}$ & $0,6 \%$ & $67 \%$ & $0,40 \%$ \\
$\mathrm{Fe}$ & $1,5 \%$ & $3 \%$ & $0,05 \%$ \\
$\mathrm{Al}$ & $2,5 \%$ & $6 \%$ & $0,15 \%$ \\
$\mathrm{Si}$ & $1,5 \%$ & $22 \%$ & $0,32 \%$
\end{tabular}

Aunque finalmente se solucionara la avería, modificando varias cosas a la vez y sin saber cual era el motivo real de la misma, la técnica de realizar el análisis por duplicado se ha mantenido. La preparación de muestras en forma de perla requiere unos 7 a 8 minutos; y el análisis por duplicado, unos 7 minutos. El tiempo total de preparación de muestra y: análisis por duplicado es de unos 15 minutos, suficiente para nuestras necesidades actuales.

Un problema que no hemos llegado aún a solucionar ha sido el análisis del Mg. Inicialmente trabajabamos con perlas 1:5, y actualmente con perlas 1:3 (muestra:bórax). En cualquiera de los casos la cantidad de muestra en la perla analizada es muy pequeña (antes $0,5 \mathrm{~g}$ y actualmente $0,75 \mathrm{~g}$ ), y en nuestro crudo sólo poseemos de 0,7 a $0,9 \%$ de $\mathrm{MgO}$. Por ello las cuentas obtenidas eran muy pocas, y la dispersión muy grande. Todo ello nos ha impedido, junto con los "saltos" del canal n. 5 que analizaba al Mg, obtener datos fiables de él.

Sin embargo, nos interesaba conocer su valor, más o menos aproximado. En primer lugar, porque proporcionamos todos los análisis de crudo en forma calcinada (como si fuera un clínker), y en forma de $\mathrm{CaO}, \mathrm{SiO}_{2}, \mathrm{Al}_{2} \mathrm{O}_{3}, \mathrm{Fe}_{2} \mathrm{O}_{3}$ y $\mathrm{MgO}$, y con suma constante a $99,50 \%$. Y, además de facilitar los módulos de saturación en cal, de sílice y de alúmina, damos la titulación en carbonato de la misma.

Hemos solucionado el problema, por el momento, considerando como valor constante del $\mathrm{MgO}$ el $2 \%$ de la $\mathrm{CaO}$ presente en la muestra. $\mathrm{Y}$ con ello, mientras usamos unas calizas y arcillas de una misma procedencia, las variaciones entre el análisis por rayos $\mathrm{X} \mathrm{y}$ por vía química tradicional nos dan diferencias en el $\mathrm{MgO}$, que en valor absoluto suelen ser inferiores a $0,10 \%$ sobre valores que oscilan entre 1,30 y $1,50 \%$ de $\mathrm{MgO}$ en las muestras analizadas. Si se utilizan materias primas distintas, debe calcularse un nuevo coeficiente.

\section{Cómo se lleva a cabo el control de calibraciones periódicas}

Hemos observado que el clínker portland, preparado en forma de perla de bórax, podía analizarse por rayos $\mathrm{X}$ utilizando la misma curva de calibración de crudos, si se tenían en cuenta las cantidades a pesar de muestra y bórax. 
Ello no es extraño, ya que el clínker se obtiene precisamente al calcinar el material crudo. Y los únicos cálculos que debían hacerse eran para determinar qué cantidad de crudo queda después de la calcinación. Un crudo que posea unos módulos análogos a los de consigna posee una pérdida al fuego de un $36 \%$; por ello, después de calcinado un crudo nos quedan $2 / 3$ del peso original del mismo. Si en una perla de crudo se pesan $0,750 \mathrm{~g}$ de crudo y 2,250 g de bórax, para preparar una perla de clínker pesamos 0,500 g de clínker y $2,250 \mathrm{~g}$ de bórax.

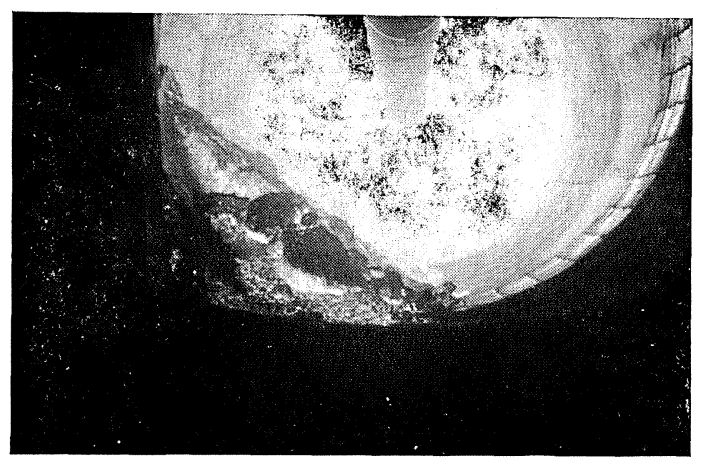

Vista interior del horno.

Aprovechando esta circunstancia, diariamente se comparan los resultados de análisis del clínker medio del día por vía química tradicional y por rayos $\mathrm{X}$. Si las diferencias entre ambos resultados son pequeñas (en módulo de saturación menor o igual al $2 \%$, y en módulos de sílice y de alúmina menores o iguales a 0,05$)$, se considera que los resultados confirman el perfecto funcionamiento del equipo. En caso contrario se investiga cuál es la causa de dicha discrepancia, y se pasan algunas muestras de crudo de análisis conocido (de las de calibración de crudo) para ver

las diferencias. Ello nos puede llevar a efectuar nueva calibración, o comprobar la discriminación.

Las perlas de las muestras de calibración se guardan al abrigo del aire, en desecadores, para evitar que absorban humedad y se deterioren. Con este única precaución permanecen inalteradas por largo espacio de tiempo, que puede llegar a 1 ó $1 \frac{1}{2}$ años.

La periodicidad de cambio de los filamentos de los detectores viene a ser de unos 6 meses. La realización de calibraciones viene motivada por cambiar el tubo de rayos X $\mathrm{o}$ su posición, cambio de los filamentos de los detectores, cambio de la discriminación, o cambio de la botella del gas de los detectores. El tubo de rayos $\mathrm{X}$, que viene funcionando a una potencia de $2,5 \mathrm{~kW}$, lo hemos venido sustituyendo cada 5.000-6.000 horas de funcionamiento.

\section{Problemas que ha causado el ordenador de proceso}

Hasta el momento presente, las averías que ha sufrido el ordenador han sido muy pocas y el servicio de asistencia de IBM nos ha atendido con suma celeridad, no debiendo dejar nunca el control del crudo por causa del ordenador.

La parte más propensa a averías es la máquina impresora de salida de datos. Pero disponiendo de otras, dedicadas a otros fines, no ha obligado nunca a parar el control del proceso. En ocasiones al realizar operaciones incorrectas se han destruido programas o ficheros de datos, debiendo en el primer caso cargar los programas destruidos y en el segundo inicializar el control a partir de los últimos datos correctos conocidos.

El servicio técnico de IBM dispone de una serie de programas de "autochequeo" del propio ordenador, mediante los cuales él mismo se detecta el lugar donde posee la avería. 


\section{ESTUDIOS DE REPRODUCIBILIDAD}

\section{Reproducibilidad del aquipo de rayos $\mathbf{X}$}

En múltiples ocasiones se ha efectuado un ensayo que nos pusiese de manifiesto la reproducibilidad del espectrómetro de rayos $\mathrm{X}$, analizando una misma muestra en forma de perla, durante 10, 20 ó 25 veces consecutivas. Con ello se puede determinar la reproducibilidad del equipo a corto plazo.

Los resultados han sido satisfactorios en la mayoría de ocasiones, y cuando no lo fueron nos pusieron de manifiesto que alguna anomalía perturbaba al equipo.

T A B L A 1

ESTUDIO DE LA REPRODUCIBILIDAD DEL EQUIPO DE RAYOS X

Análisis de una misma perla de crudo, 25 veces consecutivas.

(Los resultados se expresan como cuentas o impulsos detectados).

\begin{tabular}{lccccc}
\hline $\begin{array}{c}\text { N.o } \\
\text { muestra }\end{array}$ & $\mathbf{C a}$ & Fe & Al & Si & Mg \\
\hline 5001 & 25121 & 5999 & 2391 & 1436 & 247 \\
5002 & 24980 & 5993 & 2329 & 1444 & 245 \\
5003 & 25010 & 5994 & 2362 & 1443 & 247 \\
5004 & 25025 & 5985 & 2353 & 1447 & 243 \\
5005 & 25055 & 5999 & 2379 & 1437 & 256 \\
5006 & 25030 & 5979 & 2317 & 1434 & 251 \\
5007 & 25027 & 5998 & 2331 & 1439 & 247 \\
5008 & 25027 & 6002 & 2350 & 1445 & 248 \\
5009 & 25028 & 5993 & 2372 & 1436 & 249 \\
5010 & 25047 & 5982 & 2367 & 1443 & 247 \\
5011 & 25074 & 5997 & 2369 & 1433 & 255 \\
5012 & 25043 & 6008 & 2343 & 1448 & 246 \\
5013 & 25008 & 5984 & 2341 & 1447 & 249 \\
5014 & 25057 & 5979 & 2377 & 1443 & 247 \\
5015 & 25020 & 5992 & 2352 & 1442 & 256 \\
5016 & 25085 & 5996 & 2362 & 1437 & 244 \\
5017 & 25096 & 5994 & 2378 & 1440 & 244 \\
5018 & 25070 & 6005 & 2375 & 1442 & 251 \\
5019 & 25051 & 5998 & 2398 & 1436 & 252 \\
5020 & 25055 & 6008 & 2364 & 1432 & 250 \\
5021 & 25023 & 5992 & 2357 & 1443 & 237 \\
5022 & 25038 & 5991 & 2349 & 1446 & 247 \\
5023 & 25028 & 5994 & 2362 & 1434 & 251 \\
5024 & 25040 & 5986 & 2343 & 1441 & 247 \\
5025 & 25134 & 6006 & 2393 & 1450 & 245 \\
\hline & & & &
\end{tabular}

Como muestra de ellos, véanse las tablas núms. 1 y 2 de un estudio realizado el 4 de septiembre de 1972. Si bien son muy importantes las bajas desviaciones estándar abso- 
lutas conseguidas en cada elemento $\left(0,08 \%\right.$ en $\mathrm{CaO}$ y $\mathrm{SiO}_{2}, 0,05 \%$ en $\mathrm{Al}_{2} \mathrm{O}_{3}$ y $0,00 \%$ en $\mathrm{Fe}_{2} \mathrm{O}_{3}$ ), las referentes a módulos:

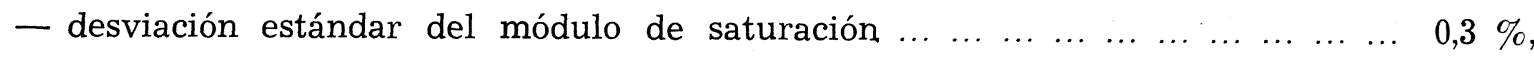

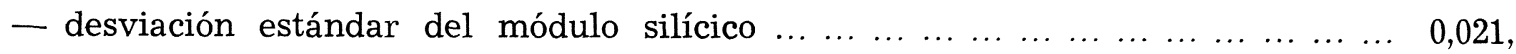

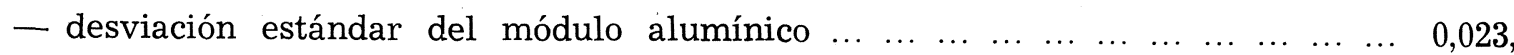

son inferiores a las máximas desviaciones estándar que nos garantizara Philips:

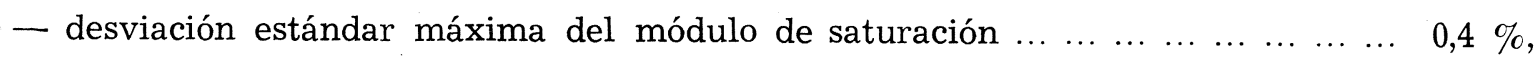

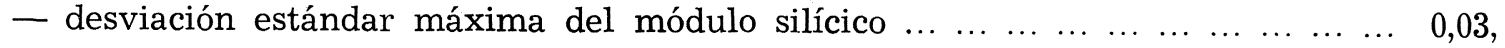

$\begin{array}{lllllllllll}\text { - desviación estándar máxima del módulo alumínico } & \ldots & \ldots & \ldots & \ldots & \ldots & \ldots & \ldots & \ldots & \ldots & 0,05 .\end{array}$

T A в L A 2

ESTUDIO DE LA REPRODUCIBILIDAD DEL EQUIPO DE RAYOS X

Análisis de una misma perla de crudo, 25 veces consecutivas.

(Los resultados se expresan como concentraciones y módulos).

\begin{tabular}{|c|c|c|c|c|c|c|c|c|c|}
\hline $\begin{array}{l}\text { N.o } \\
\text { muestra }\end{array}$ & $\mathrm{CaO}$ & $\mathbf{S i O}_{2}$ & $\mathbf{A l}_{2} \mathbf{O}_{3}$ & $\mathrm{Fe}_{2} \mathrm{O}_{3}$ & MgO & SAT. CALC. & M. SILIC. & M. ALUM. & $\mathrm{CO}_{3} \mathrm{Ca}$ \\
\hline 5001 & 0.6580 & 0.2321 & 0.0665 & 0.0266 & 0.0131 & 0.882 & 2.493 & 2.500 & 0.7601 \\
\hline 5002 & 0.6573 & 0.2320 & 0.0658 & 0.0267 & 0.0131 & 0.882 & 2.508 & 2.463 & 0.7598 \\
\hline 5003 & 0.6569 & 0.2314 & 0.0667 & 0.0266 & 0.0131 & 0.882 & 2.475 & 2.504 & 0.7596 \\
\hline 5004 & 0.6568 & 0.2319 & 0.0664 & 0.0266 & 0.0131 & 0.881 & 2.492 & 2.496 & 0.7595 \\
\hline 5005 & 0.6577 & 0.2301 & 0.0673 & 0.0266 & 0.0131 & 0.887 & 2.448 & 2.522 & 0.7602 \\
\hline 5006 & 0.6593 & 0.2303 & 0.0654 & 0.0266 & 0.0131 & 0.891 & 2.500 & 2.452 & 0.7615 \\
\hline 5007 & 0.6583 & 0.2309 & 0.0658 & 0.0267 & 0.0131 & 0.887 & 2.495 & 2.462 & 0.7606 \\
\hline 5008 & 0.6571 & 0.2316 & 0.0663 & 0.0266 & 0.0131 & 0.882 & 2.489 & 2.485 & 0.7597 \\
\hline 5009 & 0.6577 & 0.2302 & 0.0671 & 0.0266 & 0.0131 & 0.887 & 2.453 & 2.516 & 0.7602 \\
\hline 5010 & 0.6572 & 0.2311 & 0.0668 & 0.0265 & 0.0131 & 0.884 & 2.472 & 2.514 & 0.7598 \\
\hline 5011 & 0.6586 & 0.2294 & 0.0669 & 0.0266 & 0.0131 & 0.891 & 2.449 & 2.510 & 0.7609 \\
\hline 5012 & 0.6570 & 0.2320 & 0.0660 & 0.0267 & 0.0131 & 0.882 & 2.501 & 2.473 & 0.7596 \\
\hline 5013 & 0.6569 & 0.2321 & 0.0660 & 0.0266 & 0.0131 & 0.881 & 2.504 & 2.481 & 0.7596 \\
\hline 5014 & 0.6571 & 0.2309 & 0.0671 & 0.0265 & 0.0131 & 0.884 & 2.464 & 2.528 & 0.7597 \\
\hline 5015 & 0.6574 & 0.2312 & 0.0664 & 0.0266 & 0.0131 & 0.884 & 2.483 & 2.492 & 0.7599 \\
\hline 5016 & 0.6584 & 0.2300 & 0.0667 & 0.0266 & 0.0131 & 0.889 & 2.463 & 2.501 & 0.7607 \\
\hline 5017 & 0.6578 & 0.2302 & 0.0671 & 0.0266 & 0.0131 & 0.887 & 2.455 & 2.522 & 0.7602 \\
\hline 5018 & 0.6573 & 0.2307 & 0.0670 & 0.0266 & 0.0131 & 0.885 & 2.461 & 2.514 & 0.7599 \\
\hline 5019 & 0.6574 & 0.2298 & 0.0679 & 0.0266 & 0.0131 & 0.887 & 2.429 & 2.547 & 0.7599 \\
\hline 5020 & 0.6586 & 0.2295 & 0.0668 & 0.0267 & 0.0131 & 0.891 & 2.450 & 2.500 & 0.7609 \\
\hline 5021 & 0.6572 & 0.2313 & 0.0666 & 0.0266 & 0.0131 & 0.883 & 2.480 & 2.498 & 0.7598 \\
\hline 5022 & 0.6571 & 0.2317 & 0.0662 & 0.0266 & 0.0131 & 0.882 & 2.493 & 2.488 & 0.7597 \\
\hline 5023 & 0.6582 & 0.2300 & 0.0668 & 0.0267 & 0.0131 & 0.889 & 2.458 & 2.503 & 0.7606 \\
\hline 5024 & 0.6579 & 0.2310 & 0.0661 & 0.0266 & 0.0131 & 0.886 & 2.490 & 2.482 & 0.7604 \\
\hline 5025 & 0.6566 & 0.2312 & 0.0674 & 0.0265 & 0.0131 & 0.882 & 2.460 & 2.536 & 0.7593 \\
\hline MEDIO & 0.6576 & 0.2309 & 0.0666 & 0.0266 & 0.0131 & 0.885 & 2.474 & 2.500 & 0.7601 \\
\hline MINIMO & 0.6566 & 0.2294 & 0.0654 & 0.0265 & 0.0131 & 0.881 & 2.429 & 2.452 & 0.7593 \\
\hline MAXIMO & 0.6593 & 0.2321 & 0.0679 & 0.0267 & 0.0131 & 0.891 & 2.508 & 2.547 & 0.7615 \\
\hline DESV. & 0.0008 & 0.0008 & 0.0005 & 0.0000 & 0.0000 & 0.003 & 0.021 & 0.023 & 0.0008 \\
\hline
\end{tabular}


Los ensayos de reproducibilidad a largo plazo se han realizado en pocas ocasiones, y los resultados hallados han sido bastante dispares, por lo cual no es oportuno citarlos. Estamos llevando a cabo más estudios de este tipo; y de obtener resultados concordantes, ya se publicarán oportunamente.

Mejor reproducibilidad, a corto plazo, se ha obtenido al analizar durante quince análisis consecutivos una misma muestra, pero siendo cada uno el valor medio de un análisis duplicado. Ver tabla adjunta n. ${ }^{\circ} 3$.

T A B L A 3

ESTUDIO DE LA REPRODUCIBILIDAD DEL EQUIPO DE RAYOS X

Análisis de una misma perla de crudo, 15 veces consecutivas.

(Los resultados, expresados como concentraciones y módulos, corresponden cada uno individualmente a la media de dos análisis).

\begin{tabular}{lccccccccc}
\hline $\begin{array}{c}\text { N.o } \\
\text { muestra }\end{array}$ & CaO & SiO $_{2}$ & $\mathbf{A l}_{2} \mathbf{O}_{3}$ & $\mathbf{F e}_{2} \mathbf{O}_{3}$ & MgO & SAT. CALC. & M. SILIC. & M. ALUM. & CO $_{3}$ Ca \\
\hline 5002 & 0.6809 & 0.2066 & 0.0624 & 0.0313 & 0.0136 & 1.012 & 2.203 & 1.995 & 0.7788 \\
5004 & 0.6799 & 0.2076 & 0.0623 & 0.0314 & 0.0135 & 1.006 & 2.213 & 1.982 & 0.7780 \\
5006 & 0.6805 & 0.2067 & 0.0626 & 0.0314 & 0.0136 & 1.010 & 2.198 & 1.990 & 0.7785 \\
5008 & 0.6793 & 0.2073 & 0.0631 & 0.0314 & 0.0135 & 1.005 & 2.191 & 2.007 & 0.7776 \\
5010 & 0.6811 & 0.2061 & 0.0626 & 0.0314 & 0.0136 & 1.014 & 2.191 & 1.994 & 0.7790 \\
5012 & 0.6795 & 0.2075 & 0.0628 & 0.0314 & 0.0135 & 1.005 & 2.200 & 1.996 & 0.7778 \\
5014 & 0.6795 & 0.2076 & 0.0629 & 0.0313 & 0.0135 & 1.005 & 2.202 & 2.007 & 0.7777 \\
5016 & 0.6807 & 0.2064 & 0.0627 & 0.0313 & 0.0136 & 1.012 & 2.194 & 2.004 & 0.7787 \\
5018 & 0.6798 & 0.2071 & 0.0629 & 0.0314 & 0.0135 & 1.007 & 2.193 & 2.004 & 0.7780 \\
5020 & 0.6800 & 0.2067 & 0.0632 & 0.0313 & 0.0136 & 1.009 & 2.184 & 2.016 & 0.7781 \\
5022 & 0.6804 & 0.2672 & 0.0623 & 0.0313 & 0.0136 & 1.009 & 2.211 & 1.987 & 0.7784 \\
5024 & 0.6805 & 0.2077 & 0.0616 & 0.0314 & 0.0136 & 1.008 & 2.233 & 1.960 & 0.7785 \\
5026 & 0.6799 & 0.2071 & 0.0628 & 0.0314 & 0.0135 & 1.008 & 2.196 & 1.998 & 0.7781 \\
5028 & 0.6792 & 0.2073 & 0.0634 & 0.0314 & 0.0135 & 1.004 & 2.187 & 2.017 & 0.7775 \\
5030 & 0.6802 & 0.2062 & 0.0635 & 0.0314 & 0.0136 & 1.011 & 2.172 & 2.022 & 0.7783 \\
MEDI0 & 0.6801 & 0.2070 & 0.0627 & 0.0314 & 0.0136 & 1.008 & 2.198 & 1.998 & 0.7782 \\
MINIM0 & 0.6792 & 0.2061 & 0.0616 & 0.0313 & 0.0135 & 1.004 & 2.172 & 1.960 & 0.7775 \\
MAXIMO & 0.6811 & 0.2077 & 0.0635 & 0.0314 & 0.0136 & 1.014 & 2.233 & 2.022 & 0.7790 \\
DESV. & 0.0007 & 0.0005 & 0.0004 & 0.0000 & 0.0000 & 0.002 & 0.013 & 0.014 & 0.0006 \\
\hline
\end{tabular}

\section{Reproducibilidad de la muestra bajo forma de perla}

Para efectuar este estudio se ha partido de una muestra, previamente homogeneizada, de la cual se han confeccionado diez distintas perlas de bórax, las cuales se han analizado dos veces, y se dieron a conocer los resultados individuales de cada uno de dichos análisis. Finalmente se han determinado los valores máximo, mínimo y medio, así como la desviación estándar de cada una de las concentraciones y módulos. Los resultados se reflejan en la tabla $n .^{\circ} 4$. 
T A B L A 4

\section{ESTUDIO DE LA REPRODUCIBILIDAD DE LA MAQUINA DE PREPARACION DE PERLAS}

Análisis de una misma muestra de crudo, bajo forma de diez perlas distintas, analizadas cada una 2 veces consecutivas.

\begin{tabular}{cccccccccc}
\hline $\begin{array}{c}\mathbf{N} .^{\circ} \\
\text { muestra }\end{array}$ & $\mathbf{C a O}$ & $\mathbf{S i O}_{2}$ & $\mathbf{A l}_{2} \mathbf{O}_{3}$ & $\mathbf{F e}_{2} \mathbf{O}_{3}$ & Mgo & SAT. CALC. & M. SILIC. & M. ALUM. & CO $_{3} \mathbf{C a}$ \\
\hline 5001 & 0.6820 & 0.2171 & 0.0538 & 0.0283 & 0.0136 & 0.988 & 2.641 & 1.901 & 0.7797 \\
5101 & 0.6823 & 0.2174 & 0.0532 & 0.0283 & 0.0136 & 0.988 & 2.666 & 1.879 & 0.7800 \\
\hline 5002 & 0.6821 & 0.2176 & 0.0532 & 0.0282 & 0.0136 & 0.987 & 2.667 & 1.883 & 0.7798 \\
5102 & 0.6811 & 0.2177 & 0.0541 & 0.0282 & 0.0136 & 0.984 & 2.641 & 1.916 & 0.7790 \\
\hline 5003 & 0.6849 & 0.2142 & 0.0537 & 0.0283 & 0.0136 & 1.004 & 2.610 & 1.897 & 0.7821 \\
5103 & 0.6860 & 0.2132 & 0.0536 & 0.0283 & 0.0137 & 1.010 & 2.600 & 1.894 & 0.7829 \\
\hline 5004 & 0.6835 & 0.2177 & 0.0518 & 0.0281 & 0.0136 & 0.991 & 2.718 & 1.840 & 0.7809 \\
5104 & 0.6826 & 0.2170 & 0.0535 & 0.0281 & 0.0136 & 0.990 & 2.657 & 1.901 & 0.7802 \\
\hline 5005 & 0.6825 & 0.2177 & 0.0528 & 0.0282 & 0.0136 & 0.988 & 2.686 & 1.872 & 0.7801 \\
5105 & 0.6821 & 0.2178 & 0.0531 & 0.0281 & 0.0136 & 0.987 & 2.676 & 1.887 & 0.7798 \\
\hline 5006 & 0.6817 & 0.2182 & 0.0530 & 0.0282 & 0.0136 & 0.984 & 2.685 & 1.881 & 0.7795 \\
5106 & 0.6835 & 0.2158 & 0.0532 & 0.0286 & 0.0136 & 0.996 & 2.635 & 1.854 & 0.7809 \\
\hline 5007 & 0.6844 & 0.2161 & 0.0519 & 0.0287 & 0.0136 & 0.998 & 2.677 & 1.805 & 0.7816 \\
5107 & 0.6816 & 0.2176 & 0.0540 & 0.0280 & 0.0136 & 0.986 & 2.652 & 1.928 & 0.7794 \\
\hline 5008 & 0.6880 & 0.2116 & 0.0532 & 0.0281 & 0.0137 & 1.021 & 2.598 & 1.889 & 0.7845 \\
5108 & 0.6856 & 0.2136 & 0.0536 & 0.0284 & 0.0137 & 1.008 & 2.603 & 1.887 & 0.7826 \\
\hline 5009 & 0.6829 & 0.2167 & 0.0531 & 0.0284 & 0.0136 & 0.992 & 2.656 & 1.869 & 0.7805 \\
5109 & 0.6829 & 0.2168 & 0.0531 & 0.0284 & 0.0136 & 0.992 & 2.656 & 1.871 & 0.7804 \\
\hline 5010 & 0.6863 & 0.2123 & 0.0539 & 0.0286 & 0.0137 & 1.013 & 2.570 & 1.885 & 0.7831 \\
5110 & 0.6857 & 0.2128 & 0.0541 & 0.0286 & 0.0137 & 1.010 & 2.571 & 1.889 & 0.7826 \\
\hline MEDI0 & 0.6836 & 0.2159 & 0.0533 & 0.0283 & 0.0136 & 0.996 & 2.643 & 1.881 & 0.7810 \\
MINIM0 & 0.6811 & 0.2116 & 0.0518 & 0.0280 & 0.0136 & 0.984 & 2.570 & 1.805 & 0.7790 \\
\hline MAXIM0 & 0.6880 & 0.2182 & 0.0541 & 0.0287 & 0.0137 & 1.021 & 2.718 & 1.928 & 0.7845 \\
DESV. & 0.0019 & 0.0020 & 0.0006 & 0.0001 & 0.0000 & 0.010 & 0.038 & 0.026 & 0.0015 \\
\hline & & & & & & \\
5
\end{tabular}

Las desviaciones estándar como índice de las variaciones resultantes de la preparación de muestra, nos ponen de manifiesto que, en cuanto a concentraciones, son de doble magnitud que las motivadas por la propia reproducibilidad del equipo, y que, en cuanto a módulos, las desviaciones que presenta el módulo de saturación en cal es 3 veces mayor, mientras que las de los módulos de sílice y de alúmina son sólo 1,5 veces mayores.

De todas formas, los resultados obtenidos se pueden considerar como más aceptables para un buen control de crudo.

Realizados los mismos ensayos en otra ocasión, las dispersiones halladas fueron casi la mitad de las actuales. En aquella ocasión se partió de una muestra de las utilizadas para la calibración del equipo, y de ella se podía garantizar un mayor grado de homogeneidad. 
Pudiera influir, en un mayor o menor grado de dispersión de resultados, la exactitud de las pesadas de muestra y bórax. Pero los diferentes resultados que pudieran motivarse por dicha inexactitud serán comentados en el apartado siguiente.

\section{Reproducibilidad de la perla, con distintas dosificaciones fundentes}

La primera variación apreciable de resultados, en forma de cuentas, se observa al variar sustancialmente la cantidad pesada de muestra y de bórax. Hasta una variación de peso del $12 \%$ de crudo o del $5 \%$ de bórax, los resultados analíticos no varían sustan-

T A в L A 5

\section{ESTUDIO DE LA REPRODUCIBILIDAD DE LA MAQUINA DE PREPARACION} DE PERLAS

Análisis de una misma muestra de crudo, preparada a partir de distintas proporciones de crudo y bórax, y con doble análisis consecutivo.

(Los resultados se expresan como cuentas o impulsos detectados).

\begin{tabular}{|c|c|c|c|c|c|c|c|}
\hline $\begin{array}{c}\text { N.॰ } \\
\text { muestra }\end{array}$ & $\mathbf{C a}$ & $\mathbf{F e}$ & Al & $\mathbf{S i}$ & $\mathbf{M g}$ & $\begin{array}{c}\begin{array}{c}\text { Peso de } \\
\text { crudo } \\
(\mathrm{g})\end{array} \\
\end{array}$ & $\begin{array}{l}\begin{array}{c}\text { Peso de } \\
\text { bórax } \\
(\mathrm{g})\end{array} \\
\end{array}$ \\
\hline 6001 & 24115 & 7015 & 2167 & 1266 & 248 & $\mathrm{M}=0,75$ & $B=2,25$ \\
\hline 6101 & 24049 & 7012 & 2150 & 1264 & 255 & $\mathrm{M}=0,75$ & $B=2,25$ \\
\hline 6002 & 22996 & 6813 & 2008 & 1241 & 258 & $\mathrm{M}=0,70$ & $B=2,25$ \\
\hline 6102 & 23016 & 6818 & 2016 & 1243 & 251 & $\mathrm{M}=0,70$ & $B=0,25$ \\
\hline 6003 & 21836 & 6595 & 1897 & 1170 & 253 & $\mathrm{M}=0,65$ & $B=2,25$ \\
\hline 6103 & 21827 & 6591 & 1888 & 1170 & 241 & $\mathrm{M}=0,65$ & $B=2,25$ \\
\hline 6004 & 25630 & 7260 & 2320 & 1363 & 261 & $\mathrm{M}=0,80$ & $B=2,25$ \\
\hline 6104 & 25636 & 7248 & 2356 & 1360 & 254 & $\mathrm{M}=0,80$ & $B=2,25$ \\
\hline 6005 & 26146 & 7348 & 2407 & 1442 & 257 & $\mathrm{M}=0,85$ & $B=2,25$ \\
\hline 6105 & 26131 & 7366 & 2434 & 1440 & 246 & $\mathrm{M}=0,85$ & $B=2,25$ \\
\hline 6006 & 24555 & 7093 & 2233 & 1305 & 257 & $\mathrm{M}=0,75$ & $B=2,20$ \\
\hline 6106 & 24536 & 7097 & 2220 & 1298 & 251 & $M=0,75$ & $B=2,20$ \\
\hline 6007 & 24614 & 7053 & 2198 & 1349 & 256 & $\mathrm{M}=0,75$ & $\mathrm{~B}=2,15$ \\
\hline 6107 & 24528 & 7043 & 2212 & 1346 & 253 & $\mathrm{M}=0,75$ & $\mathrm{~B}=2,15$ \\
\hline 6008 & 25165 & 7215 & 2315 & 1340 & 251 & $M=0,75$ & $\mathrm{~B}=2,10$ \\
\hline 6108 & 25168 & 7222 & 2336 & 1331 & 245 & $\mathrm{M}=0,75$ & $B=2,10$ \\
\hline 6009 & 23634 & 6943 & 2112 & 1284 & 253 & $\mathrm{M}=0,75$ & $B=2,30$ \\
\hline 6109 & 23604 & 6925 & 2088 & 1279 & 253 & $\mathrm{M}=0,75$ & $B=2,30$ \\
\hline 6010 & 23214 & 6874 & 2068 & 1206 & 252 & $\mathrm{M}=0,75$ & $\mathrm{~B}=2,35$ \\
\hline 6110 & 23176 & 6864 & 2072 & 1203 & 249 & $\mathrm{M}=0,75$ & $\mathrm{~B}=2,35$ \\
\hline 6011 & 22534 & 6732 & 1985 & 1221 & 241 & $\mathrm{M}=0,75$ & $B=2,40$ \\
\hline 6111 & 22554 & 6699 & 1999 & 1223 & 243 & $\mathrm{M}=0,75$ & $B=2,40$ \\
\hline 6012 & 16450 & 5546 & 1370 & 833 & 232 & $\mathrm{M}=0,50$ & $B=2.50$ \\
\hline 6112 & 16453 & 5572 & 1388 & 834 & 242 & $M=0,50$ & $B=2,50$ \\
\hline
\end{tabular}


cialmente, si bien existe una acusada variación en el número de impulsos contabilizados. Teniendo en cuenta que los errores de pesada suelen ser como máximo del orden del $\mathrm{mg}$, ello pone de mạnifiesto que las dispersiones en los análisis de una misma muestra en forma de perlas distintas son motivadas por heterogeneidades del material crudo analizado. Ver tablas $n .^{\circ s} 5$ y 6.

T A B L A 6

\section{ESTUDIO DE LA REPRODUCIBILIDAD DE LA MAQUINA DE PREPARACION DE PERLAS}

Análisis de una misma muestra de crudo, preparada a partir de distintas proporciones de crudo $y$ bórax $y$ con doble análisis consecutivo.

(Los resultados se expresan en forma de concentraciones y módulos).

\begin{tabular}{|c|c|c|c|c|c|c|c|c|c|c|c|}
\hline $\begin{array}{c}\text { N.o } \\
\text { muestra }\end{array}$ & CaO & $\mathbf{S i O}_{2}$ & $\mathbf{A l}_{2} \mathbf{O}_{3}$ & $\mathbf{F e}_{2} \mathbf{O}_{3}$ & Mgo & s & M. SILIC. & I. & $\mathrm{CO}_{3} \mathrm{Ca}$ & $\begin{array}{l}\text { Peso de } \\
\text { crudo }\end{array}$ & $\begin{array}{l}\text { Peso de } \\
\text { bórax }\end{array}$ \\
\hline 001 & 0.6735 & 0.2147 & 0.0616 & 0.0315 & 0.0134 & 0.969 & 2.302 & 1.953 & 0.7729 & 0,75 & 2,25 \\
\hline 01 & 0.6735 & 0.2150 & 0.0612 & 0.0316 & 0.0134 & 0.969 & 2.313 & 1.936 & 0.7730 & 0,75 & 2,25 \\
\hline 02 & 0.6701 & 2202 & 0.0592 & 0.0319 & 134 & 0.947 & 2.414 & 1.8 & 70 & 0,70 & 2,25 \\
\hline 02 & 0.6698 & 0.2203 & 0.0594 & 0.0319 & 0.0133 & 0.946 & 2.410 & 1.859 & 0.7700 & 0,70 & 2,25 \\
\hline 03 & 0.6711 & 0.2188 & 0.0589 & 0.0326 & 0.0134 & 0.953 & 2.388 & 1.805 & 0.7710 & 0,65 & 2,25 \\
\hline 103 & 0.6712 & 0.2189 & 0.0586 & 0.0326 & 0.0134 & 0.954 & 2.398 & 1.796 & 0.7711 & 0,65 & 2,25 \\
\hline 04 & 0.6716 & 0.2172 & 0.0620 & 0.0306 & 134 & 0.957 & 2.342 & 2.023 & 0.7714 & 0,80 & 2,25 \\
\hline 104 & 0.6713 & 0.2165 & 0.0631 & 0.0305 & 0134 & .958 & 2.309 & 2.064 & 0.7711 & 0,80 & 2,25 \\
\hline 005 & 0.6649 & 0.2238 & 0.0626 & 0.0301 & 132 & 0.9 & 2.4 & 2.0 & 0.7661 &, 85 & 2,25 \\
\hline 05 & 0.6644 & 0.2235 & 0.0635 & 0.0301 & 0.0132 & 0.922 & 2.384 & 2.104 & 0.7656 & 0,85 & 2,25 \\
\hline & 711 & 68 & & 12 & 34 & & & & 10 & 75 & 2,20 \\
\hline 106 & 0.6720 & 0.2160 & 0.0620 & 0.0313 & 0.0134 & 0.962 & 2.314 & 1.980 & 0.7718 & 0,75 & 2,20 \\
\hline 07 & 0.6682 & 0.2217 & 07 & 0.0308 & 133 & 0 & 2. & 1 & 887 & 0,75 & 2,15 \\
\hline 107 & 0.6664 & 0.2231 & 0.0612 & 0.0308 & 0.0133 & 0.929 & 2.422 & 1 & 0.7672 & 0,75 & 2,15 \\
\hline 08 & 0.6703 & 0.2170 & 631 & & 134 & 0.954 & 2.306 & & 0.7704 & 0,75 & 2,10 \\
\hline 108 & 0.6709 & 0.2156 & 0.0638 & 0.0310 & 0.0134 & 0.959 & 2.271 & 2.059 & 0.7709 & 0,75 & 2,10 \\
\hline & 0.6680 & 12 & & & 33 & 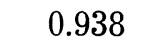 & & & 885 &, 75 & 2,3 \\
\hline 8109 & 0.6690 & 0.2208 & 0.0600 & 0.0316 & 0.0133 & 0.942 & 2.408 & 1.899 & 0.7693 & 0,75 & 2,30 \\
\hline 010 & 0.6753 & 0.2127 & 611 & 22 & 35 & 0.980 & 2.278 & 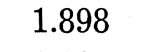 & 0.7743 & 0,75 & 2,35 \\
\hline 6110 & 0.6752 & 0.2125 & 0.0614 & 0.0322 & 0.0135 & 0.980 & 2.269 & 1.906 & 0.7743 & 0,75 & 2,35 \\
\hline 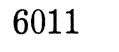 & 0 & 08 & 0 & 0 & 33 & 0 . & 2. & 1. & 92 & 0,75 & 2,40 \\
\hline 6111 & 0.6685 & 0.2209 & 0.0601 & 0.0319 & 0.0133 & 0.941 & 2.396 & 1.881 & 0.7689 & 0,75 & 2,40 \\
\hline 6012 & 0.6796 & 0.2081 & & & 135 & 1.000 & 2.225 & & 0.7778 & 0,50 & 2,50 \\
\hline 6112 & 0.6787 & 0.2081 & 0.0575 & 0.0369 & 0.0135 & 1.006 & 2.202 & 1.554 & 0.7771 & 0,50 & 2,50 \\
\hline MEDIO & 0 & & & & & & & & 99 & & \\
\hline MINIMO & 0.6644 & 0.2081 & 0.0566 & 0.0301 & 132 & 0.922 & 2.202 & & 0.7656 & & \\
\hline MAXIMO & 0.6796 & 0.2238 & 0.0638 & 0.0369 & 0.0135 & 1.008 & 2.422 & 2.104 & 0.7778 & & \\
\hline DESV. & 0.0037 & 0.0042 & 0.0018 & 0.0016 & 0.0000 & 0.021 & 0.065 & 0.139 & 0.0030 & & \\
\hline
\end{tabular}


Reproducibilidad de análisis con perlas fundidas a distinta temperatura. Refundición de perlas rotas

La temperatura de fusión, una vez se logre la franca fusión del material fundido, no tiene importancia sobre los resultados analíticos. Tampoco influye el hecho de que dicha fusión sea más o menos prolongada, dentro de determinados límites.
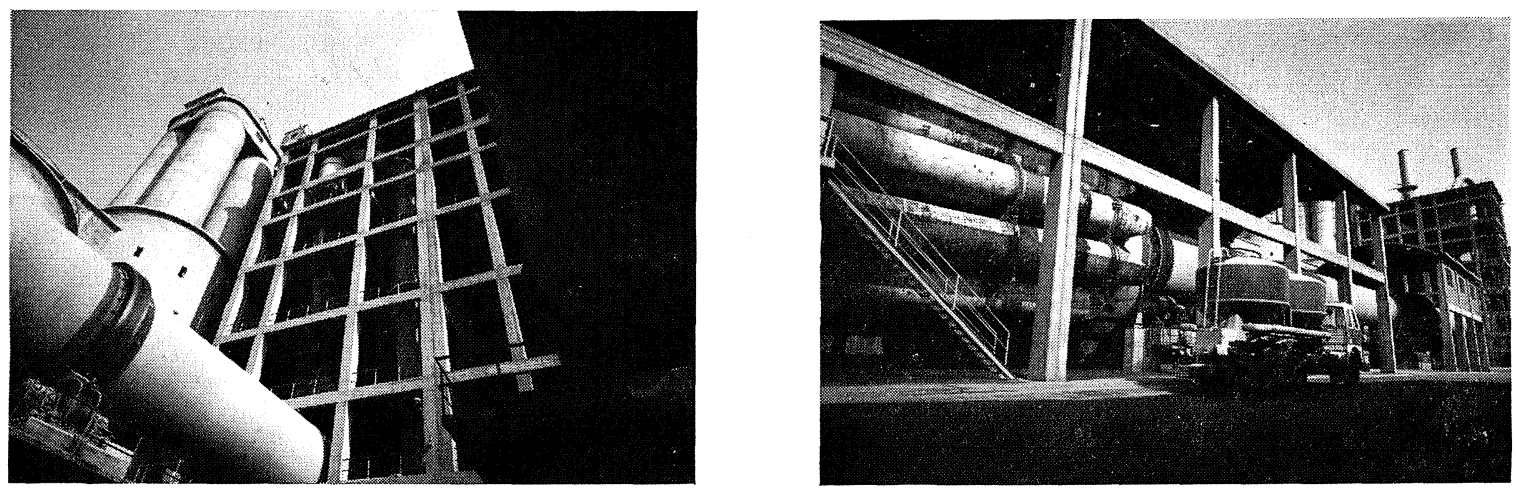

$\mathrm{Si}$, por el contrario, no se llegó a una temperatura suficientemente elevada y no se aprecia homogeneidad en la perla, los resultados son muy distintos después de fundir con muestra nueva a una temperatura más elevada o bien después de refundir la perla.

Como ejemplo de lo dicho se llevó a cabo un estudio que consiste en analizar una muestra en forma de perla, volver a fundir la perla y analizarla de nuevo, y así sucesivamente hasta quedar tan delgada que se rompía. Los resultados fueron altamente satisfactorios. Ver tabla n. ${ }^{\circ} 7$.

$$
\text { T A в L A } 7
$$

\section{ESTUDIO DE LA REPRODUCIBILIDAD DE LA MAQUINA DE PREPARACION DE PERLAS}

Análisis de una misma muestra de crudo, en forma de perla; una vez analizada, era sometida a una nueva fusión y analizada de nuevo.

Se pudo analizar 4 veces consecutivas, pero no admitió la cuarta fusión.

(Los resultados se expresan en forma de concentraciones y módulos).

\begin{tabular}{lccccccccc}
\hline $\begin{array}{c}\text { N.o } \\
\text { muestra }\end{array}$ & $\mathbf{C a O}$ & $\mathbf{S i O}_{2}$ & $\mathrm{Al}_{2} \mathbf{O}_{3}$ & $\mathbf{F e}_{2} \mathbf{O}_{3}$ & MgO & SAT. CALC. & M. SILIC. & M. ALUM. & CO $\mathbf{C a}_{3}$ \\
\hline 5001 & 0.6879 & 0.2067 & 0.0563 & 0.0301 & 0.0137 & 1.034 & 2.389 & 1.869 & 0.7844 \\
5002 & 0.6861 & 0.2078 & 0.0572 & 0.0300 & 0.0137 & 1.025 & 2.381 & 1.905 & 0.7830 \\
\hline 5003 & 0.6867 & 0.2075 & 0.0570 & 0.0299 & 0.0137 & 1.028 & 2.387 & 1.905 & 0.7834 \\
5004 & 0.6855 & 0.2090 & 0.0569 & 0.0296 & 0.0137 & 1.020 & 2.412 & 1.921 & 0.7825 \\
\hline MEDIO & 0.6865 & 0.2078 & 0.0569 & 0.0299 & 0.0137 & 1.027 & 2.392 & 1.900 & 0.7833 \\
MINIMO & 0.6855 & 0.2067 & 0.0563 & 0.0296 & 0.0137 & 1.020 & 2.381 & 1.869 & 0.7825 \\
MAXIMO & 0.6879 & 0.2090 & 0.0572 & 0.0301 & 0.0137 & 1.034 & 2.412 & 1.921 & 0.7844 \\
DESV. & 0.0009 & 0.0008 & 0.0003 & 0.0001 & 0.0000 & 0.005 & 0.012 & 0.019 & 0.0007 \\
\hline
\end{tabular}


Una experiencia análoga se hizo con una perla a la que se sometió a varias fusiones, pero en cada una de ellas se adicionaban unos $20 \mathrm{mg}$ de bórax. Las cuentas variaron sustancialmente, mientras que los resultados analíticos fueron muy similares. Ver tabla n. 8.

\section{MEJORAS CONSEGUIDAS EN EL CONTROL DE CALIDAD}

Las ventajas de este nuevo sistema de control de crudo, mediante espectrómetro de rayos $\mathrm{X}$ y ordenador de proceso, con respecto al método tradicional de titulación en carbonatos, son las siguientes:

\section{1. $\left.{ }^{a}\right)$ Mejores datos analíticos del crudo}

No cabe duda que la actual información de la muestra analizada, que nos facilita el análisis químico completo de la misma y sus módulos, es mucho más completa que la simple titulación en carbonato.

\section{2. ${ }^{\text {a) }}$ Mejor conocimiento de las materias primas utilizadas}

Con el actual sistema de control de crudo, después de cada análisis de una muestra de molienda, se dispone del análisis estadístico, bastante aproximado y orientativo, de las materias primas que utilizamos, dato que anteriormente nos era desconocido.

T A B L A 8

\section{ESTUDIO DE LA REPRODUCIBILIDAD DE LA MAQUINA DE PREPARACION DE PERLAS}

Análisis de una misma muestra de crudo, en forma de perla. Después del primer análisis, se fundía de nuevo, con adición de $20 \mathrm{mg}$ de bórax, y era nuevamente analizada.

(Los resultados se expresan en forma de cuentas o impulsos detectados, y en forma de concentraciones y módulos).

\begin{tabular}{|c|c|c|c|c|c|c|c|c|c|}
\hline $\begin{array}{c}\text { N.o } \\
\text { muestra }\end{array}$ & & $\mathbf{C a}$ & \multicolumn{2}{|c|}{$\mathbf{F e}$} & \multicolumn{4}{|c|}{$\mathbf{S i}$} & $\mathbf{M g}$ \\
\hline 6001 & & 24337 & \multicolumn{2}{|c|}{6607} & \multicolumn{2}{|c|}{2120} & \multicolumn{2}{|l|}{1303} & 254 \\
\hline 6002 & & 23162 & \multicolumn{2}{|c|}{6470} & \multicolumn{2}{|l|}{1990} & \multicolumn{2}{|l|}{1252} & 255 \\
\hline 6003 & & 22031 & \multicolumn{2}{|c|}{6249} & \multicolumn{2}{|c|}{1873} & \multicolumn{2}{|l|}{1182} & 257 \\
\hline 6004 & & 21133 & \multicolumn{2}{|c|}{6104} & \multicolumn{2}{|c|}{1806} & \multicolumn{2}{|l|}{1123} & 258 \\
\hline 6005 & & 20174 & \multicolumn{2}{|c|}{5939} & \multicolumn{2}{|c|}{1683} & \multicolumn{2}{|l|}{1069} & 249 \\
\hline $\begin{array}{c}\text { N.o } \\
\text { muestra }\end{array}$ & $\mathbf{C a O}$ & $\mathrm{SiO}_{2}$ & $\mathbf{A l}_{2} \mathbf{O}_{3}$ & $\mathbf{F e}_{2} \mathbf{O}_{3}$ & MgO & SAT. CALC. & M. SILIC. & M. ALUM. & $\mathrm{CO}_{3} \mathrm{Ca}$ \\
\hline 6001 & 0.6718 & 0.2212 & 0.0582 & 0.0302 & 0.0134 & 0.949 & 2.500 & 1.924 & 0.7716 \\
\hline 6002 & 0.6728 & 0.2199 & 0.0593 & 0.0294 & 0.0134 & 0.954 & 2.478 & 2.013 & 0.7724 \\
\hline 6002 & 0.6732 & 0.2198 & 0.0575 & 0.0307 & 0.0134 & 0.956 & 2.487 & 1.871 & 0.7727 \\
\hline 6003 & 0.6742 & 0.2178 & 0.0580 & 0.0313 & 0.0134 & 0.964 & 2.436 & 1.849 & 0.7735 \\
\hline 6005 & 0.6754 & 0.2175 & 0.0564 & 0.0320 & 0.0135 & 0.969 & 2.458 & 1.761 & 0.7745 \\
\hline MEDIO & 0.6735 & 0.2192 & 0.0579 & 0.0307 & 0.0134 & 0.958 & 2.472 & 1.883 & 0.7729 \\
\hline MINIMO & 0.6718 & 0.2175 & 0.0564 & 0.0294 & 0.0134 & 0.949 & 2.436 & 1.761 & 0.7716 \\
\hline MAXIMO & 0.6754 & 0.2212 & 0.0593 & 0.0320 & 0.0135 & 0.969 & 2.500 & 2.013 & 0.7745 \\
\hline DESV. & 0.0012 & 0.0013 & 0.0009 & 0.0008 & 0.0000 & 0.007 & 0.022 & 0.083 & 0.0010 \\
\hline
\end{tabular}


Al realizar el control a base de la titulación en carbonato, el crudo deseado sólo se podía definir a base de prefijar un carbonato de consigna, a obtener con una mezcla adecuada de caliza y arcilla.

Actualmente, el crudo de consigna viene definido por sus módulos de saturación en cal, de sílice $\mathrm{y}$ de alúmina, pudiendo conseguirse a partir de unas proporciones adecuadas de caliza, arcilla, arenisca y cenizas de pirita tostada.

\section{4. $\left.{ }^{a}\right)$ Mejores bases para la corrección de proporciones de materias primas}

Ello nos es permitido actualmente no sólo por conocer los análisis químicos de la muestra tomada del molino y de las materias primas que se utilizaron para ello, sino porque el ordenador calcula, mediante programación lineal, cuáles son las más idóneas proporciones de materias primas para el siguiente período de molienda, teniendo pára ello en cuenta múltipes factores como: toneladas de la muestra analizada; toneladas totales acumuladas; valor medio de los módulos del material en los silos de homogeneización; valores individuales, máximos y mínimos, que no deseamos sobrepasar en el análisis del crudo a obtener en el siguiente período; realizar la corrección para llegar al valor medio de consigna con mayor o menor rapidez, en función de las toneladas acumuladas; valores máximos y mínimos de cada una de las básculas dosificadoras de materias primas; precios de las materias primas, para obtener el crudo más económico; etc.

\section{5. $\left.{ }^{a}\right)$ Más rapidez y exactitud en la corrección de proporciones de las materias primas}

Al ser el propio ordenador el que modifica los potenciómetros de las básculas dosificadoras de materias primas, no existe demora ni falsas interpretaciones en dichas correcciones, como antes podía suceder entre el analista y el molinero.

Además, el propio ordenador realiza una labor de supervisión cada 2 minutos al comprobar que las proporciones de las materias primas consumidas en dicho período de tiempo son las calculadas. En caso contrario, vuelve a modificar los potenciometros hasta conseguir las proporciones previamente calculadas.

Todas estas mejoras, difíciles de precisar económicamente, nos han facilitado el proceso en los siguientes extremos:

a) Nos ha permitido definir mejor el crudo a obtener, precisando los módulos de consigna deseados y mantenerlos dentro de muy pequeñas dispersiones y obtener así un cemento más regular. 
b) Hemos podido modificar la consigna de los módulos de saturación en cal, de sílice y de alúmina, a fin de mejorar la economía del proceso y la calidad del cemento obtenido.

c) Hemos doblado el número de materias primas utilizadas. Antes sólo se empleaban caliza y arcilla, y actualmente también se emplean, además, arenisca y cenizas de pirita tostada.

d) Nos ha permitido doblar la capacidad de molienda sin variar ni la concepción ni la capacidad del sistema de homogeneización, y obtener un crudo aún más regular que antes.

e) La mayor regularidad del crudo ha mejorado el proceso de cocción en los hornos: más producción, menor consumo calorífico y mayor duración del revestimiento.

f) Nos permite conocer perfectamente cuál es el grado actual de homogeneización conseguido, en función de la dispersión de las 24 muestras horarias del crudo que alimenta a los hornos. La desviación tipo o estándar que conseguimos habitualmente es del 1 a $2 \%$ en el módulo de saturación en cal, y de 0,03 a 0,06 en los módulos de sílice y alúmina.

No podemos comparar las ventajas respecto al tradicional sistema de control de crudo, por titulación en carbonatos, por desconocer en tal período los análisis individuales de las muestras horarias de molienda o de alimentación a los hornos. 